\title{
New occurrences of Anastrepha Schiner (Diptera: Tephritidae) in the state of Minas Gerais, Brazil*
}

\section{Novas ocorrências de Anastrepha Schiner (Diptera: Tephritidae) no estado de Minas Gerais, Brasil}

\author{
Victor Dias Pirovani ${ }^{*}$ * (1) (orcid.org/0000-0002-1143-4547) \\ David dos Santos Martins² (D) (orcid.org/0000-0002-8465-3134) \\ Keiko Uramoto ${ }^{3}$ (D) (orcid.org/0000-0003-2676-0035) \\ Paulo Sérgio Fiuza Ferreira4 (D) (orcid.org/0000-0001-7165-6398)
}

ABSTRACT: Occurrences of Anastrepha barbiellinii Lima, Anastrepha connexa Lima, Anastrepha consobrina (Loew), Anastrepha kuhlmanni Lima, Anastrepha leptozona Hendel, and Anastrepha xanthochaeta Hendel are recorded for the first time in the state of Minas Gerais, Brazil. The species were captured in McPhail traps baited with hydrolyzed protein (5\%) in orchards of cultivated species and in a remnant of the Atlantic Forest in the municipality of Viçosa, Minas Gerais, or obtained from the collection of species deposited in the Regional Museum of Entomology (UFVB) of the Universidade Federal de Viçosa, Campus Viçosa, in the state of Minas Gerais.

KEYWORDS: fruit flies; tephritidae; diversity; geographic distribution.
RESUMO: Ocorrências de Anastrepha barbiellinii Lima, Anastrepha connexa Lima, Anastrepha consobrina (Loew), Anastrepha kublmanni Lima, Anastrepha leptozona Hendel e Anastrepha xanthochaeta Hendel são registradas pela primeira vez no estado de Minas Gerais, Brasil. As espécies foram capturadas em armadilhas do tipo McPhail com um atrativo alimentar de proteína hidrolisada (5\%) em três pomares de espécies cultivadas e um remanescente de Mata Atlântica no município de Viçosa, Minas Gerais, ou obtidas por meio de levantamento de espécies de Tephritidae depositadas no Museu Regional de Entomologia (UFVB) da Universidade Federal de Viçosa, Campus Viçosa, estado de Minas Gerais.

PALAVRAS-CHAVE: mosca-das-frutas; tefritídeos; diversidade; distribuição geográfica. 
Anastrepha Schiner, 1868 (Diptera: Tephritidae), is the most diverse genus of the Tephritidae family in the Neotropical region, with more than 250 species described (URAMOTO; ZUCCHI, 2010; NORRBOM; KORYTKOWSKI, 2012). Brazil has 121 species recorded (ZUCCHI; MORAES, 2008), of which 30 are reported in the state of Minas Gerais (ZUCCHI; MORAES, 2008; DUARTE et al., 2015). Some Anastrepha species have their distribution limited to tropical and subtropical forests, while others occur in all regions of Brazil (MALAVASI, 2000; ZUCCHI, 2007).

This study aimed to report new occurrence records of fruit flies in Minas Gerais. The studies were carried out in four areas: two fruit orchards in the Campus of the Universidade Federal de Viçosa (UFV) (20 ${ }^{\circ} 45^{\prime} \mathrm{S}, 42^{\circ} 51^{\prime} \mathrm{W}$, $696 \mathrm{~m} ; 20^{\circ} 45^{\prime} \mathrm{S}, 42^{\circ} 52^{\prime} \mathrm{W}, 667 \mathrm{~m}$ ), a private fruit orchard $\left(20^{\circ} 50^{\prime} \mathrm{S}, 42^{\circ} 54^{\prime} \mathrm{W}, 718 \mathrm{~m}\right)$, and a remnant of the Atlantic Forest, with 194 ha of secondary vegetation of the seasonal semideciduous forest subtype, with a high proportion of evergreen species (ALONSO, 1977), known as "Mata do Córrego do Paraíso" (2046’S, 4550’W, 600-700 m), located about $8 \mathrm{~km}$ from the UFV Campus. In addition, a survey was conducted on fruit fly species (Diptera: Tephritidae) deposited in the Regional Museum of Entomology (UFVB) at the Universidade Federal de Viçosa, Campus Viçosa.

They were captured in a McPhail trap baited with $300 \mathrm{~mL}$ of $5 \%$ hydrolyzed protein. The traps were distributed in the tree canopies of cultivated and wild fruit trees, about $1.5 \mathrm{~m}$ from the ground. Each week the material was checked with the help of a sieve and fine point tweezers. Afterward, the traps were washed, and the baits were renewed. At each procedure, fruit flies were quantified, sexed, properly labeled, and fixed in $70 \%$ alcohol for later identification.

The samples were collected between June 2007 and December 2010 with a total of 20 traps, distributed randomly and representatively in each environment, with six traps placed in UFV orchards, two in the private orchard, and twelve in the remnant of the Atlantic Forest. We collected 13,152 specimens of fruit flies of the genus Anastrepha (7,101 females and 6,051 males). Among them, three females of Anastrepha barbiellinii Lima, 1938, were captured with a frequency of $0.61 \%$; and two females of Anastrepha leptozona Hendel,
1914 , showed $0.41 \%$ of frequency in the identified species. The presence of both species was recorded for the first time in the state of Minas Gerais.

Anastrepha barbiellinii has only one known host in Brazil - Pereskia sp. (Cactaceae) (ZUCCHI; MORAES, 2008). Its occurrence in this country has been registered in the states of Mato Grosso do Sul (midwest region); Espírito Santo, Rio de Janeiro, and São Paulo (southeast region); Paraná, Rio Grande do Sul, and Santa Catarina (south region). Anastrepha leptozona has been recorded infesting 13 hosts from five different botanical families in Brazil Anacardiaceae, Icacinaceae, Myrtaceae, Rubiaceae, and Sapotaceae -, in 15 states - Acre, Amapá, Amazonas, Rondônia, Roraima, and Tocantins (north region); Bahia, Maranhão, and Piauí (northeast region); Goiás, Mato Grosso, and Mato Grosso do Sul (midwest region); Espírito Santo, Rio de Janeiro, and São Paulo (southeast region) (ZUCCHI; MORAES, 2008).

Four species of the genus Anastrepha were identified and deposited in UFVB, all without prior record in the state of Minas Gerais: Anastrepha connexa Lima, 1934 (1우, collected in X.1938), identified by Dr. Ângelo Moreira da Costa Lima, from Universidade Federal Rural do Rio de Janeiro (UFRRJ), in 1939; A. consobrina (Loew, 1873) (19, collected in IX.1938), A. kuhlmanni Lima, 1934 (5우, collected in VIII.1939) and A. xanthochaeta Hendel, 1914 (1웅 collected in III.1939), identified by Dr. Roberto Antonio Zucchi, from Escola Superior de Agronomia Luiz de Queiros, Universidade de São Paulo (USP), in 1981. Anastrepha connexa has been registered in Brazil only in the state of São Paulo and has no known host in the country (ZUCCHI; MORAES, 2008). Anastrepha consobrina has been reported in the states of Bahia, Maranhão, Espírito Santo, Rio de Janeiro, and São Paulo; A. kublmanni in Rio de Janeiro, São Paulo, and Santa Catarina; and A. xanthochaeta in São Paulo, Paraná, Rio Grande do Sul, and Santa Catarina, having known hosts in the Passifloraceae family (ZUCCHI, 1978; ZUCCHI; MORAES, 2008).

Currently, the genus Anastrepha has 30 fruit fly species present in Minas Gerais (ZUCCHI; MORAES, 2008). However, with the new occurrence recorded in this study, this number increased to 36 species (Table 1).

Table 1. Species of the genus Anastrepha (Tephritidae) registered in the state of Minas Gerais, Brazil, and additional references cited.

\begin{tabular}{lcc}
\multicolumn{1}{c}{ Species } & $\begin{array}{c}\text { Place of occurrence } \\
\text { (municipality) }\end{array}$ & References \\
\hline Anastrepha aczeli (Blanchard, 1961) & Bambuí & DUARTE et al. (2015) \\
\hline Anastrepha alveata (Stone, 1942) & $\begin{array}{c}\text { Itacarambi, Jaíba, Janaúba, } \\
\text { Nova Porteirinha }\end{array}$ & CANAL et al. (1998a); ALVARENGA et al. (2010) \\
\hline Anastrepha barbiellinii (Lima, 1938*) & Viçosa & Present study \\
\hline Anastrepha bahiensis (Lima, 1937) & Jaíba, Janaúba & CANAL et al. (1998a); ALVARENGA et al. (2010) \\
\hline
\end{tabular}


Table 1. Continuation

\begin{tabular}{|c|c|c|}
\hline Species & $\begin{array}{l}\text { Place of occurrence } \\
\text { (municipality) }\end{array}$ & References \\
\hline Anastrepha barnesi (Aldrich, 1925) & Janaúba, Nova Porteirinha & CANAL et al. ( $1998 a)$ \\
\hline Anastrepha bezzii (Lima, 1934) & Viçosa & SANTOS et al. (1993); PIROVANI et al. (2010) \\
\hline Anastrepha bistrigata (Bezzi, 1919) & Lavras, Viçosa & ZUCCHI (1978); PIROVANI et al. (2010) \\
\hline Anastrepha connexa (Lima, 1934*) & Viçosa & Present study \\
\hline Anastrepha consobrina (Loew, 1873)* & Viçosa & Present study \\
\hline Anastrepha daciformis (Bezzi, 1909) & Jaíba & CANAL et al. (1998a) \\
\hline Anastrepha dissimilis (Stone, 1942) & $\begin{array}{l}\text { Itacarambi, Jaíba, Janaúba, } \\
\text { Nova Porteirinha, Viçosa }\end{array}$ & $\begin{array}{l}\text { CANAL et al. (1998a); PIROVANI et al. (2010); } \\
\text { CAMARGOS et al. (2015) }\end{array}$ \\
\hline Anastrepha distincta (Greene, 1934) & $\begin{array}{l}\text { Jaíba, Janaúba, } \\
\text { Nova Porteirinha, Viçosa }\end{array}$ & CANAL et al. (1998a); PIROVANI et al. (2010) \\
\hline $\begin{array}{l}\text { Anastrepha entodonta Canal, } \\
\text { (Uramoto and Zucchi, 2013) }\end{array}$ & $\begin{array}{l}\text { Jaíba, Janaúba, } \\
\text { Nova Porteirinha }\end{array}$ & $\begin{array}{l}\text { CANAL et al. (2013); } \\
\text { CAMARGOS et al. (2015) }\end{array}$ \\
\hline $\begin{array}{l}\text { Anastrepha flavipennis } \\
\text { (Greene, 1934) }\end{array}$ & Jaíba & CANAL et al. (1998a) \\
\hline $\begin{array}{l}\text { Anastrepha fraterculus } \\
\text { (Wiedemman, 1830) }\end{array}$ & $\begin{array}{l}\text { Itacarambi, Itajubá, Jaíba, Janaúba, } \\
\text { Nova Porteirinha, Viçosa }\end{array}$ & $\begin{array}{l}\text { ZUCCHI (1978); CARVALHO (1988); } \\
\text { ROSSI et al. (1988); CANAL et al. (1998a, } \\
\text { 1998b); PIROVANI et al. (2010); ALVARENGA } \\
\text { et al. (2010); SOUZA et al. (2012); MACHADO } \\
\text { et al. (2012); CAMARGOS et al. (2015) }\end{array}$ \\
\hline Anastrepha furcata (Lima, 1934) & Viçosa & PIROVANI et al. (2010) \\
\hline $\begin{array}{l}\text { Anastrepha grandis } \\
\text { (Macquart, 1846) }\end{array}$ & Caldas, Viçosa & ROSSI et al. (1988); PIROVANI et al. (2010) \\
\hline $\begin{array}{l}\text { Anastrepha hadropickeli Canal } \\
\text { (Uramoto and Zucchi, 2013) }\end{array}$ & $\begin{array}{l}\text { Itacarambi, Jaíba, Janaúba, } \\
\text { Nova Porteirinha }\end{array}$ & CANAL et al. (2013); CAMARGOS et al. (2015) \\
\hline Anastrepha hambletoni (Lima, 1934) & Viçosa & ZUCCHI (1978); \\
\hline Anastrepha kuhlmanni (Lima, 1934*) & Viçosa & Present study \\
\hline $\begin{array}{l}\text { Anastrepha leptozona } \\
\text { (Hendel, 1914*) }\end{array}$ & Viçosa & Present study \\
\hline Anastrepha manihoti (Lima, 1934) & Jaíba, Viçosa & $\begin{array}{l}\text { ZUCCHI (1978); PIROVANI et al. (2010); } \\
\text { CAMARGOS et al. (2015) }\end{array}$ \\
\hline Anastrepha minensis (Lima, 1937) & Viçosa & ZUCCHI (1978); PIROVANI et al. (2010) \\
\hline Anastrepha montei (Lima, 1934) & $\begin{array}{l}\text { Belo Horizonte, Itacarambi, Jaíba, } \\
\text { Janaúba, Nova Porteirinha, Viçosa }\end{array}$ & $\begin{array}{l}\text { ZUCCHI (1978); CANAL et al. (1998a); } \\
\text { PIROVANI et al. (2010) }\end{array}$ \\
\hline $\begin{array}{l}\text { Anastrepha nigripalpis } \\
\text { (Hendel, 1914) }\end{array}$ & Nova Porteirinha & CANAL et al. (1998a) \\
\hline $\begin{array}{l}\text { Anastrepha obliqua } \\
\text { (Macquart, 1835) }\end{array}$ & $\begin{array}{l}\text { Itacarambi, Jaíba, Janaúba, } \\
\text { Nova Porteirinha, Viçosa }\end{array}$ & $\begin{array}{l}\text { CANAL et al. (1998a, 1998b); ALVARENGA et al. } \\
\text { (2010); PIROVANI et al. (2010); SOUZA et al. } \\
\text { (2012); CAMARGOS et al. (2015) }\end{array}$ \\
\hline Anastrepha pickeli (Lima, 1934) & $\begin{array}{l}\text { Jaíba, Janaúba, } \\
\text { Nova Porteirinha, Viçosa }\end{array}$ & $\begin{array}{l}\text { ZUCCHI (1978); CANAL et al. (1998a); } \\
\text { PIROVANI et al. (2010); ALVARENGA et al. } \\
\text { (2010); CAMARGOS et al. (2015) }\end{array}$ \\
\hline $\begin{array}{l}\text { Anastrepha pseudoparallela } \\
\text { (Loew, 1873) }\end{array}$ & Viçosa & PIROVANI et al. (2010) \\
\hline $\begin{array}{l}\text { Anastrepha serpentina } \\
\text { (Wiedemman, 1830) }\end{array}$ & Jaíba, Matias Barbosa, Viçosa & $\begin{array}{l}\text { ZUCCHI (1978); CANAL et al. (1998a); } \\
\text { PIROVANI et al. (2010) }\end{array}$ \\
\hline Anastrepha similis (Greene, 1934) & Bambuí & DUARTE et al. (2015) \\
\hline
\end{tabular}


Table 1. Continuation.

\begin{tabular}{lcc}
\multicolumn{1}{c}{ Species } & $\begin{array}{c}\text { Place of occurrence } \\
\text { (municipality) }\end{array}$ & References \\
Anastrepha sororcula (Zucchi, 1979) & $\begin{array}{c}\text { Itacarambi, Jaíba, Janaúba, } \\
\text { Nova Porteirinha, Viçosa }\end{array}$ & $\begin{array}{r}\text { CARVALHO (1988); CANAL et al. (1998a, } \\
\text { 1998); PIROVANI et al. (2010); ALVARENGA } \\
\text { et al. (2010); SOUZA et al. (2012) }\end{array}$ \\
\hline Anastrepha turpiniae (Stone, 1942) & Jaíba & SOUZA et al. (2012) \\
\hline Anastrepha undosa (Stone, 1942) & Itacarambi, Jaíba & CANAL et al. (1998a) \\
\hline $\begin{array}{l}\text { Anastrepha xanthochaeta } \\
\text { (Hendel, 1914*) }\end{array}$ & Viçosa & Present study \\
\hline Anastrepha zenildae (Zucchi, 1979) & Itacarambi, Jaíba, Janaúba, \\
Nova Porteirinha & CANAL et al. (1998a, 1998b); ALVARENGA et al. \\
(2010); SOUZA et al. (2012); \\
CAMARGOS et al. (2015)
\end{tabular}

*New species records in the state of Minas Gerais, Brazil.

ACKNOWLEDGMENTS: Not applicable.

FUNDING: This study was supported by Conselho Nacional de Desenvolvimento Científico e Tecnológico (CNPq) through Scientific Initiation Scholarship for the first author (Process no 135781/2009-4).

CONFLICTS OF INTEREST: The authors certify that they have no commercial or associative interest that represents a conflict of interest in connection with the manuscript.

ETHICAL APPROVAL: Not applicable.

AVAILABILITY OF DATA AND MATERIAL: The material collected in the field and identified by Dr. Keiko Uramoto (one of the authors of the manuscripts) is deposited at Escola Superior de Agricultura Luiz de Queiroz (ESALQ) and can be provided in case of contact by the interested party.

AUTHORS' CONTRIBUTIONS: Conceptualization: Pirovani, V.D.; Martins, D.S.; Ferreira, P.S.F. Data curation: Ferreira, P.S.F.; Uramoto, K. Formal analysis: Pirovani, V.D.; Martins, D.S. Funding acquisition: Martins, D.S.; Ferreira, P.S.

Investigation: Pirovani, V.D. Methodology: Pirovani, V.D.; Martins, D.S.; Ferreira, P.S.F.; Uramoto, K. Project administration: Pirovani, V.D.; Martins, D.S. Supervision: Martins, D.S.; Ferreira, P.S.F.; Uramoto, K. Validation: Pirovani, V.D.; Martins, D.S.; Ferreira, P.S.F. Visualization: Pirovani, V.D.; Martins, D.S.; Ferreira, P.S.F.; Uramoto, K. Writing — original draft: Pirovani, V.D.; Martins, D. Writing - review \& editing: Pirovani, V.D.

\section{REFERENCES}

ALONSO, M.T.A. Vegetação. In: GOLDENBERG, C. (Ed.). Geografia do Brasil. v.3. Região Sudeste. Rio de Janeiro: Diretoria de Divulgação, Centro Editorial, Centro de Serviços Gráficos do IBGE, 1977. p.91-118.

ALVARENGA, C.D.; ALVES, D.A.; SILVA, M.A.; LOPES, E.N.; LOPES, G.N. Moscas-das-frutas (Diptera: Tephritidae) em pomares da área urbana no norte de Minas Gerais. Revista Caatinga, Mossoró, v.23, n.2, p.25-31, 2010.

CAMARGOS, M.G.; ALVARENGA, C.D.; GIUSTOLIN, T.A., OLIVEIRA, P.C.C.; RABELO, M.M. Moscas-das-frutas (Diptera: Tephritidae) em cafezais irrigados no Norte de Minas Gerais. Coffee Science, Lavras, v.10, n. 1, p.28-37, 2015.
CANAL, N.A.; ALVARENGA, C.D.; ZUCCHI, R.A. Análise faunística de espécies de mosca-das-frutas (Dip., Tephritidae) em Minas Gerais. Scientia Agricola, Piracicaba, v.55, n. 1, p. 15-25, 1998a. https://doi.org/10.1590/S0103-90161998000100004

CANAL, N.A.; ALVARENGA, C.D.; ZUCCHI, R.A. Níveis de infestação de goiaba por Anastrepha zenildae Zucchi (Diptera: Tephritidae), em pomares comerciais do Norte de Minas Gerais. Anais da Sociedade Entomológica do Brasil, Londrina, v.27, n.4, p.657-661, 1998b. https://doi.org/10.1590/ S0301-80591998000400021

CANAL, N.A.; URAMOTO, K.; ZUCCHI, R.A. Two new species of Anastrepha Schiner (Diptera, Tephritidae) closely related to 
Anastrepha pickeli Lima. Neotropical Entomology, v.42, p.5257, 2013. https://doi.org/10.1007/s13744-012-0091-3

CARVALHO, R.P.L. Alternativas de controle: métodos culturais, atraentes, resistência vegetal e controle biológico. In: ENCONTRO SOBRE MOSCAS-DAS-FRUTAS, 1., 1988, Campinas. Anais... Campinas: Fundação Cargil, 1988. p.86-107.

DUARTE, P.A.S.; GARCIA, F.R.M.; ANDALÓ, V. Anastrepha aczeli Blanchard and Anastrepha similis Greene (Diptera: Tephritidae) in Minas Gerais, Brazil. Ciência Rural, Santa Maria, v.45, n. 10, p.17271728, 2015. https://doi.org/10.1590/0103-8478cr20140998

MACHADO, D.L.M.; STRUIVING, T.B.; SANTOS, D.; SOUZA, S.A.S.; SIQUEIRA, D.L. Levantamento de moscas-das-frutas e seus parasitoides em citros, no município de Viçosa, Minas Gerais. Revista Ceres, Viçosa, v.59, n.6, p.877-880, 2012. https://doi. org/10.1590/SO034-737X2012000600020

MALAVASI, A. Áreas livres ou de baixa prevalência. In: MALAVASI, A.; ZUCCHI, R.A. (Orgs.). Moscas-das-frutas de importância econômica no Brasil: conhecimento básico e aplicado. Ribeirão Preto, Holos, 2000. p.175-181.

NORRBOM, A.L.; KORYTKOWSKI, C.A. New species of Anastrepha (Diptera: Tephritidae), with a key for the species of the megacantha clade. Zootaxa, Auckland, v.3478, n.3478, p.510-552, 2012. https://doi.org/10.11646/zootaxa.3478.1.43

PIROVANI, V.D.; MARTINS, D.S.; SOUZA, S.A.S.; URAMOTO, K.; FERREIRA, P.S.F. Moscas-das-frutas (Diptera: Tephritidae), seus parasitoides e hospedeiros em Viçosa, Zona da Mata Mineira. Arquivos do Instituto Biológico, São Paulo, v.77, n.4, p.727-733, 2010.

ROSSI, M.M.; MATIOLI, J.C.; BUENO, V.H.P. Principais espécies de moscas-das-frutas (Diptera: Tephritidae) e sua dinâmica populacional em pessegueiros na região de Caldas, Sul de Minas Gerais. Revista de Agricultura, Piracicaba, v.63, p.329-342, 1988.

SANTOS, G.P.; ANJOS, N.; ZANÚNCIO, J.C.; ASSIS JR., S.L. Danos e aspectos biológicos de Anastrepha bezzi Lima, 1934 (Diptera, Tephritidae) em sementes de Sterculia chicha St. Hill (Sterculiaceae). Revista Brasileira de Entomologia, v.37, n.1, p.15-18, 1993.

SOUZA, A.R.; LOPES-MIELEZRSKI, G.N.; LOPES, E.N.; QUERINO, R.B.; CORSATO, C.D.A.; GIUSTOLIN, T.A.; ZUCCHI, R.A. Hymenopteran parasitoids associated with frugivorous larvae in a Brazilian Caatinga-Cerrado Ecotone. Environmental Entomology, v.41, n.2, p.233-237, 2012. https://doi.org/10.1603/EN11121

URAMOTO, K.; ZUCCHI, R.A. New species of Anastrepha Schiner (Diptera: Tephritidae) from remnant area of the Atlantic Rain Forest and surroundings in the state of Espírito Santo, Brazil. Zootaxa, Auckland, v.2535, n.1, p.49-60, 2010. https://doi. org/10.11646/zootaxa.2535.1.3

ZUCCHI, R.A. Taxonomia das espécies de Anastrepha Schiner, 1868 (Dip., Tephritidae) assinaladas no Brasil. 1978. $105 \mathrm{f}$. Thesis (Doctorate in Entomology) - Escola Superior de Agricultura Luiz de Queiroz, Universidade de São Paulo, Piracicaba, 1978. https://doi.org/10.11606/T.1 1.2019. tde-20191220-105903

ZUCCHI, R.A. Diversidad, distribución y hospederos del género Anastrepha en Brasil. In: HERNÁNDEZ-ORTIZ, V. (Ed.). Moscas de la fruta en Latinoamerica (Diptera: Tephritidae): diversidad, biología y manejo. Distrito Federal, México: S y G Editores, 2007. p.77-100.

ZUCCHI, R.A.; MORAES, R.C.B. Fruit flies in Brazil - Anastrepha species their host plants and parasitoids. 2008. Available from: www.lea.esalq.usp.br/anastrepha/. Access on: Apr. 292019. 